\section{Latente Tuberkulose: Screening und Therapie bei Asylbewerbern in den Niederlanden}

Spruijt I et al. Latent tuberculosis screening and treatment among asylum seekers: a mixed methods study. Eur Respir J 2019; doi:10.1183/ 13993003.00861-2019

In den Niederlanden werden Asylbewerber im Rahmen eines RöntgenThorax-Screenings auf Tuberkulose (TB) untersucht, wenn sie aus Ländern mit hoher TB-Inzidenz kommen. Damit sind bestehende pulmonale TB-Erkrankungen identifizierbar. Die aktuelle Praxis soll möglicherweise auf ein Screening für latente TB-Infektion (LTBI) umgestellt werden. Ineke Spruijt et al. untersuchten die Machbarkeit und Effektivität des LTBIScreenings und der Therapie.

Das LTBI-Screening wurde Asylbewerbern ab 12 Jahren angeboten, die aus Ländern mit einer TB-Inzidenz > 200/100000 Einwohnern stammten und aktuell in niederländischen Asylbewerberzentren wohnten. Acht Public-Health-Dienstleister führten das Screening von November 2016 bis Dezember 2017 durch. Es umfasste gezielte Informationen, einen durch die teilnehmenden Asylbewerber beantworteten Gesundheitsfragebogen und Blutproben. Nachdem akute TB ausgeschlossen und eine LTBI-Diagnose bestätigt waren, wurde den Teilnehmern eine kostenlose 3-monatige Kombinationstherapie mit Isozianid und Rifampicin angeboten. Die Therapie wurde von spezialisierten Krankenschwestern unterstützt. Mit halbstrukturierten Interviews erfassten die Untersucher die Dimensionen Ansprechbarkeit, Akzeptanz, Verfügbarkeit, Unterkunft, Bezahlbarkeit und Eignung. An diesen Interviews nahmen die TB-Pflegenden der Public-Health-Dienstleister teil sowie Teilnehmer aus Eritrea, da sie die größte Asylbewerbergruppe bildeten.

\section{Screening und Therapie: persönlich wichtig, insgesamt effektiv}

Insgesamt nahmen von 1136 eingeladenen 719 Asylbewerber an dem LTBIScreening teil. Die überwiegend männlichen Teilnehmer waren 18-34 Jahre alt, kamen zu 72\% aus Eritrea, hatten ein niedriges Bildungsniveau und waren allein in den Niederlanden. Mit der LTBITherapie begannen $84 \%$ der Teilnehmer, von denen $87 \%$ die Behandlung abschlossen.

Aus den Interviews ging u. a. hervor, dass die meisten Teilnehmer das Screening für sich persönlich und die öffentliche Gesundheit als wichtig empfanden. Zu den Bedingungen, die die Aufnahme des LTBI-Screenings förderten, gehörten persönliche und mündliche Informationsvermittlung über TB und LTBI, die Unterstützung durch professionelle Übersetzer, eine bedarfsgerechte Therapieunterstützung und die Zusammenarbeit zwischen Asylbewerberzentren, Public-Health-Dienstleistern und Kommunen. Faktoren, die das Screening und die Therapie erschwerten, waren Fahrten zu Public-Health-Dienstleistern, Sprachbarrieren und der Transport von den Asylbewerberzentren zu kommunalen Ärzten während der Therapie.

\section{FAZIT}

Insgesamt wurde das LTBI-Screening und die Therapie als realisierbar und wirksam bewertet. Voraussetzungen sind nach Einschätzung der niederländischen Arbeitsgruppe ein hoch qualitatives Versorgungsangebot mit kultur- und sprachsensitiver Informationsvermittlung zu TB und LTBI und die Kooperationen zwischen den Partnerorganisationen.

Matthias Manych, Berlin 